\title{
The preliminary investigation of potential response biomarkers to air pollution (PAHs) exposure on childhood asthma
}

\section{Yuling Bao}

Nanjing Medical University First Affiliated Hospital

Rui Jin

Nanjing Children's Hospital Affiliated to Nanjing Medical University

Jinye $\mathrm{Hu}$

Nanjing Medical University

Zhe Sun

Nanjing Medical University

Mingyan Xue

Nanjing Medical University

\section{Zilong Bian}

Nanjing Medical University

Zhang Zhan

Nanjing Medical University

Feng Chen

Nanjing Medical University

Lei Li

Nanjing Medical University qian Wu ( $\nabla$ wuqian@njmu.edu.cn )

Nanjing medical university https://orcid.org/0000-0001-5155-2428

\section{Research article}

Keywords: Asthma, air pollution, PAHs, microbiota, metabolome

Posted Date: February 17th, 2020

DOI: https://doi.org/10.21203/rs.2.23723/v1

License: (1) (1) This work is licensed under a Creative Commons Attribution 4.0 International License.

Read Full License 


\section{Abstract}

\section{Background}

In recent five years, air quality in China has been gradually improved; however, the prevalence of asthma in China has still increased significantly. This study aims to examine whether air pollution exposure is a significant contributor to childhood asthma and to explore the mechanisms using exposure and response biomarkers.

\section{Methods}

The generalized additive model was applied to evaluate the effects of air pollutants on asthma hospitalization. Urinary 1-hydroxypyrene (1-OHPyr) was determined as PAHs exposure biomarker. Metabolic profiles and microbial diversity were screened and selected as response biomarkers.

Results

Daily average concentrations of the six common air pollutants were not significantly associated with daily childhood asthma hospitalization. Urinary 1-OHPyr level showed large variability among asthmatic children and were positively correlated with the abundance of Prevotella (genus level) and purine metabolism.

\section{Conclusions}

The biomarker of PAHs exposure is associated with metabolic and microbiological biomarkers of childhood asthma. As the potential health risk, we suggest that PAHs should be included in the national monitoring system aiming to reduce the exposure.

\section{Introduction}

Asthma is a complex disease, which is affected by both genetic and environmental factors. Epidemiological studies have shown the links between air pollution exposure and the increased prevalence and severity of asthma [1-3]. However, according to China's Environmental Status Bulletin (2014-2017), $\mathrm{PM}_{2.5}$ concentrations in the Yangtze River Delta was decreased by $34.3 \%$ from 2013 to 2017. Air quality has displayed an improving trend, while the prevalence of asthma is still on the rise[4]. At the same time, a few cohort studies have reported no significant association between air pollution and asthma prevalence $[5,6]$. Contradictory results bring questions regarding any changes in the role of air pollution in the prevalence of asthma [7]. Until now, there has been no update evidence regarding the association between air pollution exposure and childhood asthma in recent five years in China.

In addition, polycyclic aromatic hydrocarbons (PAHs) as the most abundant component of atmospheric particulate matters has been found related to the onsets of asthma or exacerbation of asthma symptoms [8-12]. Recent evidence suggests that PAHs exposure altered the abundance of commensal bacteria that 
were associated with health outcomes. Atmospherica PAHs exposure could influence the composition and abundance of skin microbiota and induce endocrine signaling pathway disrupted [13]. It has been demonstrated that the development of asthma is highly associated with the microbiogical circumstance[14]. Atmospherica PAHs exposure may also induce the aberrant metabolic profiling focused on oxidative stress that leads to airway inflammation[15]. Importantly, studies in childhood asthma linking environmental PAH exposure, human commensal microbiota and metabolic pathways are largely unknown. To better understand how PAHs exerts its effects on childhood asthma, omics analysis based on microbiome and metabolome were performed.

Therefore in the present study, we firstly used the public data to analyze the asscociation between the six common air pollutants $\left(\mathrm{SO}_{2}, \mathrm{NO}_{2}, \mathrm{CO}, \mathrm{O}_{3}, \mathrm{PM}_{2.5}\right.$, and $\left.\mathrm{PM}_{10}\right)$ and childhood asthma hospitalization in Nanjing for 2015-2018. We aimed to clarify the effects of these air pollutants on asthma under current environment. Then by measuring urinary 1-OHPyr as internal exposure biomarker to PAHs, we expected to find response biomarkers based on the changes of the global metabolic signatures and throat microbial diversity in asthmatic children and explored the underlying mechanisms associated with the PAHs exposure.

\section{Methods And Materials}

\subsection{Data collection}

Nanjing is the provincial capital of Jiangsu Province, China and located in Yangtze River Delta region. Children's Hospital of Nanjing Medical University and Jiangsu Women and Children Health Hospital of Nanjing Medical University are the two primary pediatric hospitals in Nanjing. It should be noted that the Nanjing Yearbook of Hygiene only reports children's hospitalization data from these two children's hospitals. Data of asthmatic hospitalized cases from January 1st, 2015 to December 31, 2018 were obtained from the Department of Respiration in the Children's Hospital of Nanjing Medical University and Jiangsu Women and Children Health Hospital of Nanjing Medical University, which were diagnosed based on the International Classification of Diseases 10th revision. Air pollution data, including daily average concentrations of $\mathrm{SO}_{2}, \mathrm{NO}_{2}, \mathrm{CO}, \mathrm{O}_{3}, \mathrm{PM}_{2.5}$, and $\mathrm{PM}_{10}$, were obtained from China National Environmental Monitoring Center. Relative humidity, mean temperature, and wind speed were obtained from China Meteorological Administration. Generalized additive model was applied to evaluate the effects of each air pollutant $\left(\mathrm{SO}_{2}, \mathrm{NO}_{2}, \mathrm{CO}, \mathrm{O}_{3}, \mathrm{PM}_{2.5}\right.$, and $\left.\mathrm{PM}_{10}\right)$ on asthma hospitalization. To suppress the potential confounding effect, firstly, we introduced a natural cubic smooth function of calendar time was introduced to exclude the weather confounding effects. We also included the indicator variable for "day of the week (DOW)" and "holiday" in the model. In addition, we stratified the data by gender and age group. Further, we used single-pollutant model to explore the effects of air pollution on asthma with single lag days ( $\operatorname{lag} 0,1,2,3,4,5,6)$ and multiple lag days ( $\operatorname{lag} 0-1,0-2,0-3,0-4,0-5$, and $0-6)$. The estimated effect of a pollutant was evaluated by the Relative Risk (RR) and its $95 \%$ confidence interval $(\mathrm{Cl})$ in daily asthma per unit concentration increased. All the analyses were performed in R software 
(version 3.5.3) with the mgcv package. All tests were two-sided, and $\mathrm{P}<0.05$ were considered statistically significant.

\subsection{Sample Collection}

The samples consisted of 20 asthmatic subjects and 20 healthy control subjects were collected from the Children's Hospital of Nanjing Medical University in April 2018. The Nanjing Medical University Clinical Research Ethics Committee, Nanjing, China, reviewed and approved the protocols of this study. Informed consent was obtained from the participants for the use of samples in this study. The clinic physician collected a throat swab and a morning urine sample from each asthmatic or healthy control subject. The swab and urine samples were stored at $-80^{\circ} \mathrm{C}$ until analysis.

\subsection{Detection of 1-OHPyr in urine by UPLC- Orbitrap -MS}

For each urine sample, $1 \mathrm{ml}$ of urine was added with $500 \mu \mathrm{L}$ of $0.2 \mathrm{M}$ sodium acetate $(\mathrm{pH}=5)$ and $5 \mu \mathrm{L}$ of $\beta$-glucuronidase with sulfatase (Helix pomatia-H2, G0876), and then incubated at $37^{\circ} \mathrm{C}$ for $2 \mathrm{~h}$. After deconjugation, the sample was centrifuged at 10,000 rpm for $10 \mathrm{~min}$, and the supernatant was collected and extracted by dichloromethane. The combined organic phase was dried under nitrogen and reconstituted in $100 \mathrm{ml}$ of $\mathrm{CH}_{3} \mathrm{OH}$. The final solution was analyzed for 1-OHPyr on an ultra-performance liquid chromatography - Orbitrap-mass spectrometry (UPLC-Orbitrap-MS) system (Thermo Fisher Scientific, Bremen, Germany). A volume of $20 \mu \mathrm{L}$ was loaded into the Hypersil Gold C18 column (1.9 $\mu \mathrm{m}$, $2.1 \times 100 \mathrm{~mm}$ ) and analyzed by linear gradient elution. The mobile phase was methanol and ultrapure water. In the linear gradient elution, $5 \%$ water linearly increased to $95 \%$ in the first 8 min and restored equilibrium back to $5 \%$ water from $8.5 \mathrm{~min}$. The flow rate was $3 \mathrm{ml} / \mathrm{min}$ and the temperature of the column was set at $35^{\circ} \mathrm{C}$. The mass spectrometry was adopted the heating $\mathrm{ESI}$ and the negative ion mode. The spray ion voltage, gas pressure of sheath and the auxiliary gas were $+3.5 \mathrm{kV}, 35$ arbitrary unit, and 10 arbitrary unit, respectively. Besides, the flow rate of $\mathrm{N}_{2}$ was $10.0 \mathrm{~L} / \mathrm{min}$ and the atomization temperature and capillary temperature was $350^{\circ} \mathrm{C}$ and $263^{\circ} \mathrm{C}$, respectively. The scan range was set $100-$ $300 \mathrm{~m} / \mathrm{z}$. The 1-OHPyr level was adjusted from urine creatinine, which was determined by a Hitachi 7100 automatic biochemical analyzer.

\subsection{Microbiota Diversity By 16s rRNA Gene Sequencing}

The total DNA was extracted by the E.Z.N.A. ${ }^{\circledR}$ soil kits (Omega Bio-tek, Norcross, GA, USA) following the manufacturer's instruction. The V3-V4 variable region of $16 \mathrm{~s}$ rRNA gene was amplified by universal primers (338F: 5'-ACTCCTACGGGAGGCAGCAG-3'; 806R: 5'-GGACTACHVGGGTWTCTAAT-3')(ABI GeneAmp ${ }^{\circledR}$ 9700). PCR products were purified with AxyPrep DNA Gel Extraction Kit (Axygen Biosciences, Union City, CA, USA). The DNA library was constructed using TruSeq ${ }^{\text {TM }}$ DNA Sample Prep Kit. Sequencing was performed on the Miseq PE300 (Illumina, San Diego, USA) platform. The raw sequence was assembled by the Fast Length Adjustment of Short reads (FLASH) software after quality filtering by Trimmomatic software. Chimeras were removed by UCHIME. OTUs was generated with $97 \%$ similarity using UPARSE (version $7.1 \mathrm{http}: / /$ drive5.com/uparse/). OTUs was illustrated according to RDP classifier 
(http://rdp.cme.msu.edu/), mapping with Silva database(SSU128), with a confidence threshold of $70 \%$. Diversity analysis was performed. Potential biomarkers analysis were identified using Wilcoxon rank-sum test to determine the significant differences between the asthmatic and control groups. PICRUSt was used to predict a profile of putative pathways from the 16S rRNA OTU data classified accoding to the Greengenes Database. KEGG (Kyoto Encyclopaedia of Genes and Genomes) database was used to categorize pathways. All data were analyzed on the free online platform of Majorbio I-Sanger Cloud Platform (www.i-sanger.com, Shanghai Majorbio Bio-pharm Technology Co., Ltd.)

\subsection{Metabolic profiles by UPLC- Orbitrap -MS}

The urinary samples were pretreated by methanol $(1: 3, \mathrm{~V} / \mathrm{V})$. The supernatant was prepared for further analysis after centrifuged at $10000 \mathrm{rpm} / \mathrm{min}$. The analyses were also performed on the above UPLCOrbitrap-MS system. The protocal was clearly described in our previous work [16]. In brief, A multistep gradient consisting of $0.1 \%$ formic acid in water $(A)$ and $0.1 \%$ formic acid in acetonitrile (B) was applied. The UPLC autosampler temperature was set at $4^{\circ} \mathrm{C}$ and the injection volume was $10 \mu \mathrm{l}$. The operating parameters of the MS were set up as follows: spray voltage of $3 \mathrm{kV}$, capillary temperature of $300^{\circ} \mathrm{C}$, and flow of the sheath gas, auxiliary gas, sweep gas, and S-Lens RF level were at 40, 10, 2, and 50 arbitrary units, respectively. In the full scan analyses (70 to $1050 \mathrm{amu}$, amu: atomic mass unit), the resolution was set at $7 \times 10^{5}$ with an automatic gain control target of $1 \times 10^{6}$ charges, and a maximum injection time of $120 \mathrm{~ms}$. The mass spectrometry was calibrated every $24 \mathrm{~h}$ to ensure mass accuracy.All the raw data files were preprocessed using the SIEVE software (Thermo Fisher Scientific, Bremen, Germany) for further multivariate statistical analyses. A supervised analyses, partial least squares discriminant analyses (PLSDA) was then performed to optimize classification and search for variables by the SIMCA-P 13.0 software package. The variable importance in the projection (VIP) was obtained from the PLS-DA model, which indicates a relative contribution of each variable on the classification, and VIP $>1.0$ were and $P$-value $<0.05$ were considered statistically significant as potential biomarkers.

\section{Result}

\subsection{No correlation between asthma and six criteria air pollutants}

In this study, children's asthma hospitalization peaked in late spring (from March to May) and fall (from September to November), as shown in Fig. 1. Bronchial asthma attack often occurs in the spring and fall [17]. The arrival of spring brings pollen allergy from grasses, weeds, and trees, and the fall means the transition to the cold days, which are common triggers of asthma attacks. There was a gender difference in childhood asthma (STable 1). Male children were significantly more likely to be sensitized to allergens [17]. Figure 2 showed that there was no significant association between the six common air pollutants, except for lag0 of $\mathrm{SO}_{2}$, lag3 of $\mathrm{NO}_{2}$, lag3 of $\mathrm{O}_{3}$ and lag0 of $\mathrm{PM}_{10}$. Subgroup analysis by gender and age showed no significant effects. The comparison of the trend pattern between the month average concentrations of six-air pollutant and asthma hospitalization was shown in Sfig.1. Except for $\mathrm{O}_{3}$, the peaks of $\mathrm{SO}_{2}, \mathrm{NO}_{2}, \mathrm{CO}, \mathrm{PM}_{2.5}$, and $\mathrm{PM}_{10}$ concentrations were ahead of that of asthma hospitalization. 
Although it is not statistically significant, it still indicated that after the peak of $\mathrm{O}_{3}$ concentration, there was an increase in asthma-related hospital admissions. The monthly average relative humidity, temperature and average wind speed also matched with the hospital admissions, as shown in Sfig.2; however there was still no significant correlation .

\subsection{1-OHPyr Levels In Urine Samples}

In this study, the characteristics of the subjects being sampled were described in Table 1. All the asthmatic children adminstrated control medicine, such as inhaled corticosteroid (ICS), long-acting beta agonists (LABAs), short-acting beta agonists (SABAs), oral anti-allergic medicine, and immunomodulator. asthmatic and control subjects had healthy diets with fried or grilled food intake at 0-1 time/week, were living in the city, lived within 2 kilometers from schools, walked or were sent by electric bike to school, lived in homes with vent-out kitchens, and had non-smoking parents. The urinary 1-OHPyr level ranged from non-detected (ND) to $2.477 \mathrm{ng} / \mathrm{ml}$ in the asthma group and from ND to $0.156 \mathrm{ng} / \mathrm{ml}$ in the control group. The creatinine correction average value was $0.219 \pm 0.33 \mu \mathrm{mol} / \mathrm{mol}$ in asthmatic children and $0.137 \pm 0.052 \mu \mathrm{mol} / \mathrm{mol}$. The geometric means of two groups were not significantly different. The coefficient of variation (COV) of 1-OHPyr concentrations in the asthma group was $150.5 \%$ (standard Deviation: 0.330 , mean: 0.219$)$, and was $38.4 \%(0.052,0.137)$ in the control group. There were individual differences with the exposure levels of PAHs in the asthma group. In addition, the geometric mean of 1OHPyr levels in the National Health and Nutrition Examination Survey (NHANES) 2003-2004 subsample 6-11 year old children was 119 (in $\mathrm{ng} / \mathrm{g}$ of creatinine), that was $0.05 \mu \mathrm{mol} / \mathrm{mol} \mathrm{Cr}$ [18]. Compared with American children 1-OHPyr exposure level, the exposure level of 1-OHPyr of children in Nanjing was much higher.

Table 1

The characteristics of the subjects in this study

\begin{tabular}{|lll|}
\hline Variable & Asthma group(history of allergy) & Control group \\
\hline male & $13(7)$ & 13 \\
\hline female & $7(3)$ & 7 \\
\hline Age (yrs) & $6.45 \pm 2.3$ & $7.23 \pm 1.5$ \\
\hline Medication (\%) & \\
\hline ICS/LABA & 100 & - \\
\hline SABA & 15 & - \\
\hline Singulair & 10 & - \\
\hline Anti-allergy medicine & 20 & - \\
\hline Immunomodulator & 50 & - \\
\hline
\end{tabular}


3.3 1-OHPyr was associated with children's commensal microbiota in the throat

After sequencing, a total of 2417101 sequences were produced after optimization, and 681 OTUs were obtained based on the $97 \%$ threshold. After classified and alignment with Silva database, the OTU table contained taxonomic information with 23 phylum levels and 346 species levels. Pan analysis, rankabundance curve, and sob index were used to study the diversity of microbial communities in children with asthmatic or healthy children. The results in Sfig.3 showed that the microbial diversity and richness of the control group was more abundant than that of the asthmatic group. Rarefaction curve almost tended to approach an asymptote that reflected a perfect estimate of sampling.

The compositions of the two groups had remarkable similarity. The taxonomic composition of the microbial communities at the phylum level in asthmatic group and the control was shown in Fig. 3A and $B$, which mainly consisted of Firmicutes, Bacteroidetes, Proteobacteria, Actinobacteria, Fusobacteria and so on, and at genus level, mainly consisted of Streptococcus, Veillonella, Prevotella, Neisseria, Haemophilus, Actinomyce, Leptotrichia and so on (Fig. 3C and D). The significant differences in microbial communities were performed using the Wilcoxon rank-sum test. At the phylum level, the abundance of Proteobacteria was decreased $(P=0.0461)$ in the asthmatic group, and that firmicutes $(P>0.05)$ and Bacteroidetes $(P=0.0238)$ was increased. At the genus level, the abundance of Veillonella $(P=0.0159)$ and Prevotella $(P=0.0066)$ was increased (Fig. 4), and that of Neisseria and Haemophilus was decreased $(P>0.05)$ in asthma group. Multiple biological pathway, such as cancer, environmental adaptation, nucleotide metabolism and lipid metabolism were predicted from the throat metagenome by PICRUSt(Figure 5), and the detailed pathway was illustrated in Sig.4. Pearson correlation coefficient was applied to investigate the association between 1-OHPyr and microbiota diversity. There was some positive correlation between microorganism and 1-OHPyr level. Stomatobaculum $\square r=0.80, P<0.0001 \rrbracket$, Selenomonas $\varangle r=0.73, P<0.0001 \rrbracket$, and Prevotella $\square r=0.65, P<0.0001 \rrbracket$ was associated with higher PAHs concentration. Then a redundancy analysis (RDA) was further performed to determine the multivariate relationship between microbiota in samples and the environmental variable-PAHs by R. Although Stomatobaculum and Selenomonas was associated with PAHs level, their abundance were very low. The RDA confirmed Prevotella was positively correlated with 1-OHPyr level(Fig. 6).

\subsection{1-OHPyr are associated with metabolic profile in asthmatic children}

We also detected the metabolic profiles in asthmatic and the control group by UPLC-Orbitrap-MS. The variables with both VIP score $>1.0$ and P-value $<0.05$ were identified as candidate biomarkers. The metabolites that were confidently and putatively identified in the samples were listed in STable 2. After mapping to KEGG, the results showed that most of differential expressed metabolites (DEMs) were involved in purine metabolism, amino acid metabolism and lipid and fatty acid metabolism. Purine metabolic pathway was significantly aberrant $(P<0.0001$, Fig. 7), which was in consistent with the results from studies [19, 20]. Phenylacetaldehyde, L-3-Phenyllactic acid, Hippuric acid, 4-Hydroxybenzoic acid, and L-Tyrosine were aberrant, especially the accumulation of Phenylacetaldehyde, L-3-Phenyllactic acid and Hippuric acid, which indicated the activation of phenylalanine metabolism $(P<0.005)$. Hypotaurine, 
acting as antioxidant, involved in organic osmolytes, was increased in asthmatic group. These implied responses to the oxidative stress conditions induced by environmental factors [21]. Interestingly, the level of 2-hydroxyfluorene(2-OHFlu), the metabolite of fluorene, one of PAHs, in asthmatic group was 1.29 times than that in the control $(\mathrm{VIP}=1.06, \mathrm{P}=0.005)$. Lipid and fatty acid metabolism was also obviously influenced shown in STable 3. Phospholipid metabolites such as CL, PC, PG, and PS was increased, decreased was PE and PIP2. Phospholipids are the major component of pulmonary surfactant, attenuating the surface tension of the alveolus. Lysophospholipids in the lung were increased as pulmonary inflammation occurred and involved in the development of pneumonia and asthma [22]. TG, DG, MG, free fatty acids was increased, but Linoleic acid, polyunsaturated fatty acid, PUFA was decreased. It has been shown that PUFAs have significant roles in inflammatory process on different diseases. Our data was consistent with [23], suggesting a weakened anti-inflammatory effect. In addition, valproylglycine, prolylglycine, valyl-hydroxyproline glycylprolylhydroxyproline was increased in the asthmatic group, which were the major components of collagen-derived dipeptides and may be risk markers of osteoporosis and bone injury associated with corticosteroid treatment. Reinke et al found that prolylhydroxyproline was correlated with inhaled corticosteroid [19]. It is noted that me melatonin metabolite was very lower in the asthmatic group than that in the control group. The activation of the immune system may lead to free radical production, which may be exacerbated by PAHs exposure, associated with decreased melatonin levels in inflammatory diseases, such as allergic disease, asthma[24]. We conducted RDA analysis between 1-OHPyr and metabolites (Fig. 8). Pearson correlation showed that 1-OHPyr was positively correlated with deoxyadenosine monophosphate $(r=0.86, P<$ 0.0001), which is involved in purine metabolism $(\mathrm{VIP}=1.04$, Fold change $=1.46)$.

\section{Discussion}

By the end of 2013, the air quality-monitoring gauge $\mathrm{PM}_{2.5}$ in most cities of China have allowed to release real-time $\mathrm{PM}_{2.5}$ data. The new index standard has been fully implemented nationwide in 2016. In recent five years, due to PM management strategies and the control of power plant emissions of sulfur dioxide, monitored levels of $\mathrm{PM}_{2.5}, \mathrm{PM}_{10}$ and $\mathrm{SO}_{2}$ generally decreased the most in Yangtze River Delta Region, but $\mathrm{NO}_{2}, \mathrm{CO}$, and $\mathrm{O}_{3}$ are still at a high level. In our study, we did not find out the significant association between these six common air pollutants and asthma hospitalization. However, the pattern that $\mathrm{O}_{3}$ affected the prevalence of asthma was other than that of $\mathrm{SO}_{2}, \mathrm{NO}_{2}, \mathrm{CO}, \mathrm{PM}_{2.5}$, and $\mathrm{PM}_{10}$, which may increase the risk for asthma [25]. The numerous inconsistencies in epidemiological data may be indirect evidence of no association between air pollutants and the prevalence of childhood asthma [26]. The role of air pollution on the prevalence of asthma is weakened? The effects of air pollution on asthma may be masked by other, more influential seasonal triggers, such as infections or allergies [7]. Pollen counts usually peaks in April-May and September-October in NANJING [27]. The peak of asthma hospitalization shown in our study was consistent with these trend. What's more, even if air quality has been significantly improved recently, the accumulated effects of environmental pollution still exsit, leading to the prevalence of asthma not to decline. 
The three times national survey on asthma prevalence among children aged 0-14 years in China administered in 1990, 2000, and 2010, respectively. Three previous surveys have found that the prevalence of asthma was increasing. A new round of national survey on asthma prevalence will be coming soon. Due to the increasing of school absence rate and home burden, we should still make effort to find out the factors that exacerbates asthma. Inheritance of asthma is something that we can't stop, but we can lower risk factors to prevent from asthma exacerbation. Except for particulate matter, PAHs is also an important fossil combustion by-product. Studies have demonstrated that exposure to trafficderived PAHs may account for some of the increased asthma prevalence. Gu et al analyzed the data from the National Health and Nutrition Examination Survey (NHANES) 2001-2008 and 2011-2012 to show that a positive association between urinary 1-OHPyr and the school-age children asthma risk [28]. A recent systematic review of prenatal exposure to air pollutants on childhood wheezing and asthma reported an overall random-effects risk estimate $(95 \% \mathrm{Cl})$ was $1.04(0.94-1.15)$ for PAHs, which were focused on the 0-6 years of age[29]. These contradictory results may be due to the exposure window of PAHs, or the different measurement of PAHs levels, such as cord blood and urine sample test, land-use regression and inverse distance weight models. Moreover, in some studies there was no clear distinction between the effects of prenatal exposure from post exposure [30]. For now, China has not nation-wide monitored personal PAHs exposure level. We still lack official data to systematic evaluate the effects of the PAHs exposure on childhood asthma. Although our study did not find the difference of 1-OHPyr level between the asthmatic group and the control by targeted UPLC-MS method, the average 1-OHPyr level in Nanjing children was higher than that in USA children. What's more, the 2-OHFlu level was increased in asthmatic group by untargeted UPLC-MS method. There was a trend towards elevated PAHs, but the data were not sufficiently robust to conclude on any real association. Earlier studies primarily performed exposure assessments and toxicological studies of PAHs with human cell lines or animals at the concentration other than real serum or urine level. Such in vitro and in vivo analysis may not be sufficient to provide detailed information on how PAHs affect the cells on a molecular level. Compared with occupational PAHs exposures to high levels, the change of serum and urine level in general population is on small scale exposure to lower level. So it is important to investigate the effect of PAHs on human health at low level.

Recently the use of omics methods has been recommended in exposomics studies to identify the link between exposures and health outcomes [31]. Therefore, in this study, we used omics methods to identify potential microbiota and metabolites as putative intermediate biomarkers linking PAHs exposures to asthma outcome with plausible exposures-related pathways. In this study, Firmicutes, Bacteroidetes, and Proteobacteria (in phylum level), and Streptococcus, Neisseria, Veillonella, Haemophilus, and Prevotella (in genus level)were mainly detected in the collected throat swab, which were consistent with previous studies [32,33]. The composition of Veillonella and Prevotella was increased, and potential pathogen, Neisseria and Haemophilus was decreased in the asthmatic group. However, it was reported that compared with the control, Proteobacteria (Haemophilus) was increased and Bacteroidetes (Prevotella) was decreased in asthmatic subject with adults (37 yrs) and children (11 yrs) [34]. In our study, Prevotella and Veillonella spp. were more common in asthmatic subjects than that in the control 
individuals. Pearson correlation and RDA analysis showed that 1-OHP level was significantly positively correlated with the abundance of Prevotella (in genus level). Prevotella are Gram-negative anaerobes belonged to colonized bacteria in the normal oral, which have been found in high abundance in the lungs of children (<2yrs) with cystic fibrosis [35]. However, in present study, Proteobacteria was significantly decreased. We thought that the usage of control medicine might mask the change of microbe composition induced by asthma. Alternatively, these changes of the oral microbe composition may be due to the misuse of antibiotics. These give us an indication that medicine reversed the PAHs -induced microbiota shift related asthma. Alterations in environment-host microbiome can be an important component explaining why PAH pollution is connected to asthma.

Metabolomics can reflect the mechanism of metabolic reactions induced by environmental stressors through comparing the profiles of metabolomes in organisms. A significant variation in metabolites was observed between the asthmatic group and the control. The multivariate and pathway analysis showed there were more significant alterations in metabolic profiles between the asthmatic group and the control, suggesting a possible metabolic flux redistribution. Amino acids such as Isoleucylproline, L-beta-aspartylL-leucine, Leucyl-Alanine, Phosphoserine, Glutaminylisoleucine, Methionyl-Cysteine, L-Tyrosine, L-Alanine and Glutamic acid were decreased. Glutamic acid is used by cells to generate glutamate which is subsequently used to fuel TCA cycle [36]. The decrease of Glutamic acid and increase of Ribose 1phosphate and purine metabolites demonstrated that the metabolic stress response are capable of counteracting oxidative stress by redirecting the metabolic flux from glycolysis to pentose phosphate pathway, and then purine metabolism. N-gamma-Glutamylglutamine was increased and beneficial to glutathione synthesis via promoting the uptake of cysteine, one of the main contributors of GSH synthesis. What' more, some of DEMs enrichment in cysteine and methionine metabolism was aberrant in the study $(P<0.05)$. The serine-glycine and active methionine, Sadenosylmethionine (SAM) are onecarbon unit donor. One carbon metabolism is known for its essential role in nucleic acids synthesis, and antioxidant defense systems via generation of NADPH. One-carbon unit, methyl group, is donated by SAM. The metabolites in asthmatic group were found to have elevated levels of methylated metabolites. In these DEMs, 8-Hydroxy-7-methylguanine and 7-Methylguanine and m-Aminobenzoic acid, involved in folic acid metabolism indicated that PAHs exposure may induce DNA methylation alteration. Stressful environmental conditions, such as PAHs exposure, may lead the cells increased oxidative stress, such as oxidative DNA damage. We conclude that urine metabolomics could possibly serve as the link to trace air pollutants exposure to oxidative stress through purine metabolism, phenylalanine metabolism and onecarbon metabolism.

There are limitations to the present study. Since the subjects included in this study were all treated, these changes of microbiome and metabolome maybe resulted from the pathogenies of asthma or medicine administration. Since nasal and throat microorganisms are susceptible to environment, whether the status of colonized bacteria in the upper respiratory tract can represent the status of bacteria in the lower respiratory tract (where acute inflammation occurs in asthma) remains to be further studied. Because the number of cases in this study is small, there is no stratified analysis of PAHs in vivo exposure and asthma occurrence. Later we will increase the sample size for further analysis. Bacterial population may 
produce metabolites that interact with the host and influence the development of asthma. Although some biological pathway, such as purine metabolism, Cysteine and methionine metabolism, fatty acid biosynthesis and one carbon metaoblism, were predicted from the throat metagenome by PICRUSt, the effects of the microbiome on asthma and the potential functional mechanism should be further elucidated. Thus, our results suggested that the risk assessment of PAHs shaping commensal microbiota and metabolome profiles may need to take account into the development of microbial-metabolic interaction, which will potentially impair the human host homeostasis.

\section{Conclusion}

Six national supervised air pollutant indices has no association with the prevalence of children's asthma in the studied population from 2015-2018. The internal exposure 1-OHPyr was no significant change, but 2-OHFlu level in the asthmatic group was higher than that in the control. PAHs related biomarker showed changes in throat microbial profiles that Prevotella was positively correlated with 1-OHPyr level, and in urine metabolic profiles that the DEMs were involved in purine metabolism, phenylalanine metabolism and one-carbon metabolism, which is linked to oxidative DNA damage. We shed light on preliminary indication of changes in microbiome and metabolome upon PAHs exposure (Fig. 9). however, further investigations are required to confirm our results. At the same time, we recommend to national-wide supervise the pollution level of polycyclic aromatic hydrocarbons.

\section{Abbreviations}

1-hydroxypyrene: 1-OHPyr; polycyclic aromatic hydrocarbons: PAHs; relative risk: RR; confidence interval: Cl; ultra-performance liquid chromatography - Orbitrap-mass spectrometry : UPLC-Orbitrap-MS; Kyoto Encyclopaedia of Genes and Genomes: KEGG; operational taxonomic units: OTUs; partial least squares discriminant analyses: PLS-DA; variable importance in the projection: VIP; redundancy analysis: RDA; 2hydroxyfluorene: 2-OHFlu; Cardioplipin: CL; Phosphatidycholine: PC; Phosphatidyglyerol: PG; Phosphatidyserine: PS; Phosphatidyethanolamine: PE; phosphatidylinositol biphosphate: PIP2;.

Triglyceride: TG; diacylglycerol: DG; Monoglyceride: MG; polyunsaturated fatty acid: PUFA; differential expressed metabolites: DEMs; tricarboxylic acid: TCA; glutathione: GSH; Sadenosylmethionine: SAM.

\section{Declarations}

\section{Ethical Approval and Consent to participate}

The Nanjing Medical University Clinical Research Ethics Committee, Nanjing, China, reviewed and approved the protocols of this study. Written informed consent was obtained from the participants'parent for the use of samples in this study.

\section{Consent for publication}




\section{Availability of data and material}

Not applicable

\section{Competing Interests}

There are no conflicts to declare.

\section{Funding}

This work was supported by the National Natural Science Foundation of China (81728018 and 81530088); Natural Science Foundation of Jiangsu Province (BK20161571), Natural Science Foundation of the Higher Education Institution of Jiangsu Province (16KJA330002) and the open project of the key laboratory of modern toxicology of ministry education Nanjing Medical University (NMUAMT201802). The funders had no role in the study design, data collection and analysis, decision to publish, or preparation of the manuscript.

\section{Author Contribution}

Conceptualization, Lei Li and Qian Wu; Formal analysis, Zhe Sun, Mingyan Xue and Zilong Bian; Funding acquisition, Feng Chen and Qian Wu; Investigation, Jinye Hu, Zhe Sun, Mingyan Xue and Zilong Bian; Resources, Yuling Bao, Jin Rui, Zhan Zhang, Feng Chen and Lei Li; Writing - original draft, Qian Wu.

\section{Acknowledgements}

Not applicable

\section{References}

1. Hehua Z, Qing C, Shanyan G, Qijun W, Yuhong Z. The impact of prenatal exposure to air pollution on childhood wheezing and asthma: A systematic review. Environ Res 2017, 159:519-530.

2. Khreis H, Kelly C, Tate J, Parslow R, Lucas K, Nieuwenhuijsen M. Exposure to traffic related air pollution and risk of development of childhood asthma: A systematic review and meta-analysis. Environ Int 2017, 100:1-31.

3. Zhang Y, Ni H, Bai L, Cheng Q, Zhang H, Wang S, Xie M, Zhao D, Su H. The short-term association between air pollution and childhood asthma hospital admissions in urban areas of Hefei City in 
China: A time-series study. Environ Res $2018,169: 510-516$.

4. [Third nationwide survey of childhood asthma in urban areas of China]. National cooperation group on childhood asthma. Chin J Pediatr. 2013,51冈10冈:729-735.

5. Mölter A, Simpson A, Berdel D, Brunekreef B, Custovic A, Cyrys J, de Jongste J, de Vocht F, Fuertes E, Gehring U, Gruzieva O, Heinrich J, Hoek G, Hoffmann B, Klümper C, Korek M, Kuhlbusch TA, Lindley S, Postma D, Tischer C, Wijga A, Pershagen G, Agius R. A multicentre study of air pollution exposure and childhood asthma prevalence: the ESCAPE project. Eur Respir J 2015,45:610-624.

6. Krefis AC, Fischereit J, Hoffmann P, Pinnschmidt H, Sorbe C, Augustin M, Augustin J. Temporal analysis of determinants for respiratory emergency department visits in a large German hospital.BMJ Open Respir Res 2018,5(1):e000338.

7. Chen BY, Chen $\mathrm{CH}$, Chuang YC, Wu YH, Pan SC, Guo YL. Changes in the relationship between childhood asthma and ambient air pollution in Taiwan: Results from a nationwide survey repeated 5 years apart. Pediatr Allergy Immunol.2019,30(2):188-194.

8. Miller RL, Garfinkel R, Lendor C, Hoepner L, Li Z, Romanoff L,Sjodin A, Needham L, Perera FP, Whyatt RM. Polycyclicaromatic hydrocarbon metabolite levels and pediatric allergy and asthma in an innercity cohort. Pediatr Allergy Immunol 2010, 21:260-267.

9. Sara L Gal, Elizabeth M Noth, Jennifer Mann, John Balmes, S Katharine Hammondlra B Tager. Polycyclic aromatic hydrocarbon exposure and wheeze in a cohort of children with asthma in Fresno, CA. Journal of Exposure Science and Environmental Epidemiology 2012,22: 386-392

10. Rogula-Kozłowska, W.; Kozielska, B.; Klejnowski, K. Concentration, origin and health hazard from fine particle-bound PAH at three characteristic sites in Southern Poland. Bull Environ Contam Toxicol 2013, 91:349-355.

11. Al-Daghri NM, Alokail MS, Abd-Alrahman SH, Draz HM, Yakou SM, Clerici M. Polycyclic aromatic hydrocarbon exposure and pediatric asthma in children: a case-control study, Environ Health 2013,12: 1.

12. Karimi P, Peters KO, Bidad K, Strickland PT. Polycyclic aromatic hydrocarbons and childhood asthma. Eur J Epidemiol 2015,30(2):91-101.

13. Roslund MI, Rantala S, Oikarinen S, Puhakka R, Hui N, Parajuli A, Laitinen OH, Hyöty H, Rantalainen $A L$, Sinkkonen A; ADELE team. Endocrine disruption and commensal bacteria alteration associated with gaseous and soil PAHcontamination among daycare children. Environ Int 2019,130:104894.

14. Bisgaard H, Hermansen MN, Buchvald F, Loland L, Halkjaer LB, et al. Childhood asthma after bacterial colonization of the airway in neonates. N Engl J Med 2007,357: 1487-1495

15. Chen CS, Yuan TH, Shie RH, Wu KY, Chan CC. Linking sources to early effects by profiling urine metabolome of residents living near oil refineries and coal-fired power plants. Environ Int 2017,102:87-96.

16. Cao S, Wang L, Zhang Z, Chen F, Wu Q, Li L. Sulforaphane-induced metabolomic responses with epigenetic changes in estrogen receptor positive breast cancer cells. FEBS Open Bio. 2018,8(12):2022-2034. 
17. Eder W, Ege MJ, von Mutius E. The asthma epidemic. N Engl J Med 2006,355: 2226-2235.

18. The Fourth National Report on Human Exposure to Environmental Chemicals. CDC Centers for Disease Control and Prevention, USA. 2009: 455-457.

19. Reinke SN, Gallart-Ayala H, Gómez C, Checa A, Fauland A, Naz S, Kamleh MA, Djukanović R, Hinks TS, Wheelock CE. Metabolomics analysis identifies different metabotypes of asthma severity. Eur Respir J 2017,49(3). pii: 1601740.

20. Yu M, Cui FX, Jia HM, Zhou C, Yang Y, Zhang HW, Ding G, Zou ZM. Aberrant purine metabolism in allergic asthma revealed by plasma metabolomics. J Pharm Biomed Anal 2016,120: 181-189.

21. Orhan H, Vermeulen NP, Tump C, Zappey H, Meerman JH. Simultaneous determination of tyrosine, phenylalanine and deoxyguanosine oxidation products by liquid chromatography-tandem mass spectrometry as non-invasive biomarkers for oxidative damage. J Chromatogr B Anal Technol Biomed Life Sci 2004,799:245-254.

22. Shaikh SR, Fessler MB, Gowdy KM. Role for phospholipid acyl chains and cholesterol in pulmonary infections and inflammation. J Leukoc Biol 2016,100:985-997.

23. Zhang SY, Shao D, Liu H1, Feng J, Feng B, Song X, Zhao Q, Chu M, Jiang C, Huang W, Wang X. Metabolomics analysis reveals that benzo[a]pyrene, a component of PM2.5, promotes pulmonary injury by modifying lipid metabolism in a phospholipase A2-dependent manner in vivo and in vitro. Redox Biol 2017,13:459-469.

24. Lucia Marseglia,Gabriella D'Angelo1,Sara Manti,Carmelo Salpietro,Teresa Arrigo,Ignazio Barberi,Russel J. Reiter, Eloisa Gitto. Melatonin and Atopy: Role in Atopic Dermatitis and Asthma. Int J Mol Sci 2014,15(8):13482-13493.

25. Zu K, Liu X, Shi L, Tao G, Loftus CT, Lange S, Goodman JE. Concentration-response of short-term ozone exposure and hospital admissions for asthma in Texas. Environ Int 2017,104:139-145.

26. Hehua Z, Qing C, Shanyan G, Qijun W, Yuhong Z. The impact of prenatal exposure to air pollution on childhood wheezing and asthma: A systematic review. Environ Res 2017,159:519-530.

27. Zhang J, Xu X, Zhang ZX, Zhang Q, Yan SF. [Allergic pollen characteristics in air and its relationship with meteorological factors in NANJING]. Journal of Meteorology and Environment 2009,25(5):6771.

28. Liu H, Xu C, Jiang ZY, Gu A. Association of polycyclic aromatic hydrocarbons and asthma among children 6-19 years: NHANES 2001-2008 and NHANES 2011-2012. Respir Med $2016,110: 20-27$.

29. Hehua Z, Qing C, Shanyan G, Qijun W, Yuhong Z. The impact of prenatal exposure to air pollution on childhood wheezing and asthma: A systematic review. Environ Res 2017,159:519-530.

30. Jerzynska J, Podlecka D, Polanska K, Hanke W, Stelmach I, Stelmach W.

Prenatal and postnatal exposure to polycyclic aromatic

hydrocarbons and allergy symptomsin city children. Allergol Immunopathol (Madr) 2017,45(1):18-24.

31. Vineis P, van Veldhoven K, Chadeau-Hyam M, Athersuch TJ. Advancing the application of omicsbased biomarkers in environmental epidemiology. Environ Mol Mutagen 2013,54(7):461-467. 
32. Rogers GB, Shaw D, Marsh RL, Carroll MP, Serisier DJ, Bruce KD. Respiratory microbiota: addressing clinical questions, informing clinical practice. Thorax 2015,70(1):74-81.

33. Chotirmall SH, Gellatly SL, Budden KF, Mac Aogain M, Shukla SD, Wood DL, Hugenholtz P, Pethe K, Hansbro PM. Microbiomes in respiratory health and disease: An Asia-Pacific perspective. Respirology 2017,22(2):240-250.

34. Hilty M, Burke C, Pedro H, Cardenas P, Bush A, Bossley C, Davies J, Ervine A, Poulter L, Pachter L, et al. Disordered microbial communities in asthmatic airways. PLoS One 2010,5:e8578.

35. Zemanick ET, Wagner BD, Robertson CE, Ahrens RC, Chmiel JF, Clancy JP, Gibson RL, Harris WT, Kurland G, Laguna TA, McColley SA, McCoy K, Retsch-Bogart G, Sobush KT, Zeitlin PL, Stevens MJ, Accurso FJ, Sagel SD, Harris JK. Airway microbiota across age and disease spectrum in cystic fibrosis. Eur Respir J 2017,50(5). pii: 1700832.

36. Nissen JD, Lykke K, Bryk J, Stridh MH, Zaganas I, Skytt DM, Schousboe A, Bak LK, Enard W, Pääbo S, Waagepetersen HS. Expression of the human isoform of glutamate dehydrogenase, hGDH2, augments TCA cyclecapacity and oxidative metabolism of glutamate during glucose deprivation in astrocytes.Glia 2017,65(3):474-488.

\section{Supplementary Figure Legends}

Figure S1. The pattern match between the trend of six air pollutants and the hospital admission of asthma from 2015 to 2018.

Figure S2. The pattern match between different temperature $\left({ }^{\circ} \mathrm{C}\right)$, average relative humidity $(\%)$ and average wind speed $(\mathrm{m} / \mathrm{s})$ and the hospital admission of asthma from 2015 to 2018 .

Figure S3. The diversity indices of asthmatic group and the control A: Species richness at the OTU level; B: Rank-Abundance; C: sobs index; D: The rarefaction curves based on the sob index of OTU level.

Figure S4. The different 16S rRNA OTU enriched on the detailed KEGG pathway. The enriched pathway of the metagenome based on 16S rRNA data was performed by PICRUSt. The results were presented by STAMP. Differences between groups were determined by Wilcoxon rank-sum test.

\section{Figures}




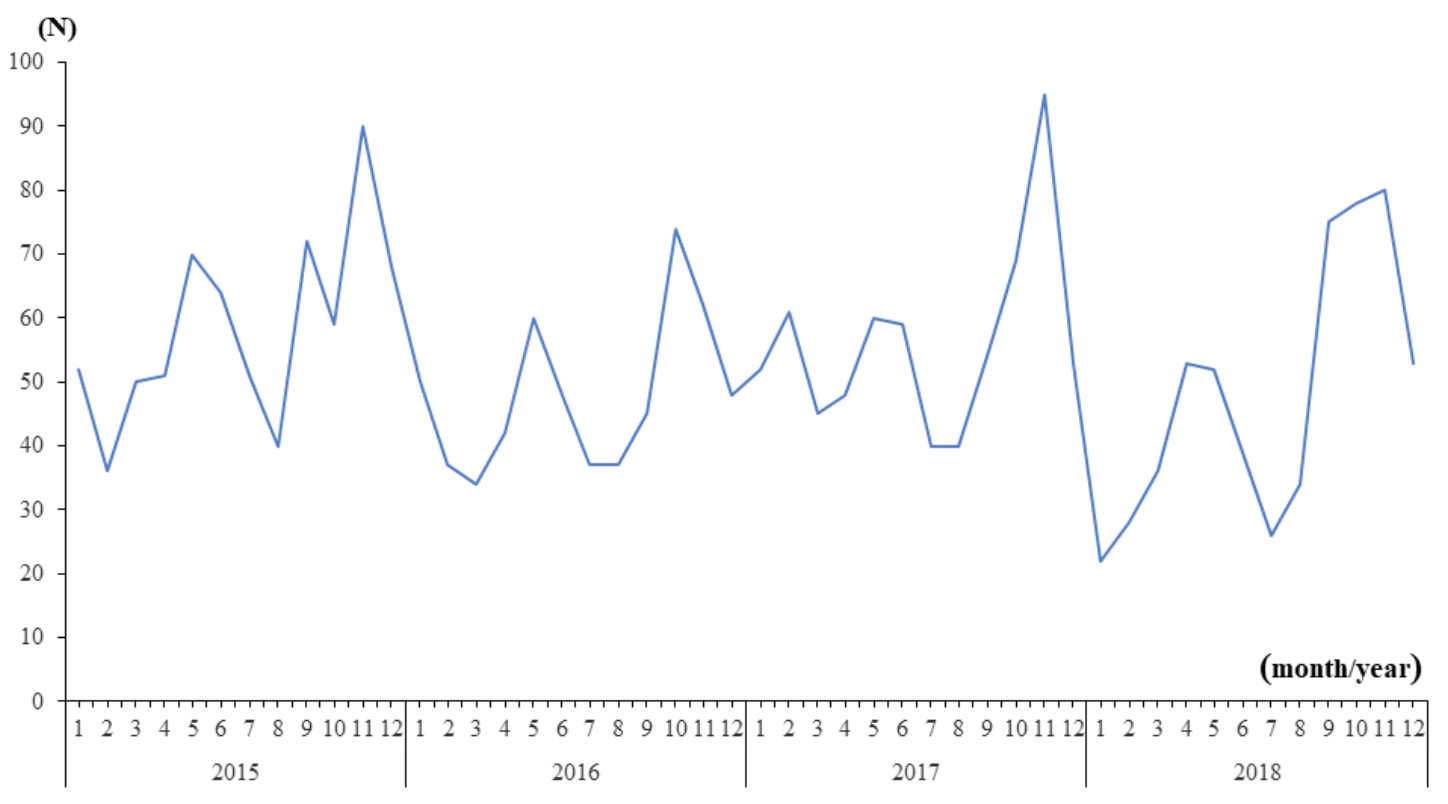

Figure 1

Daily hopitalized children due to asthma onset from 2015 to 2018.

$\mathrm{SO}_{2}$
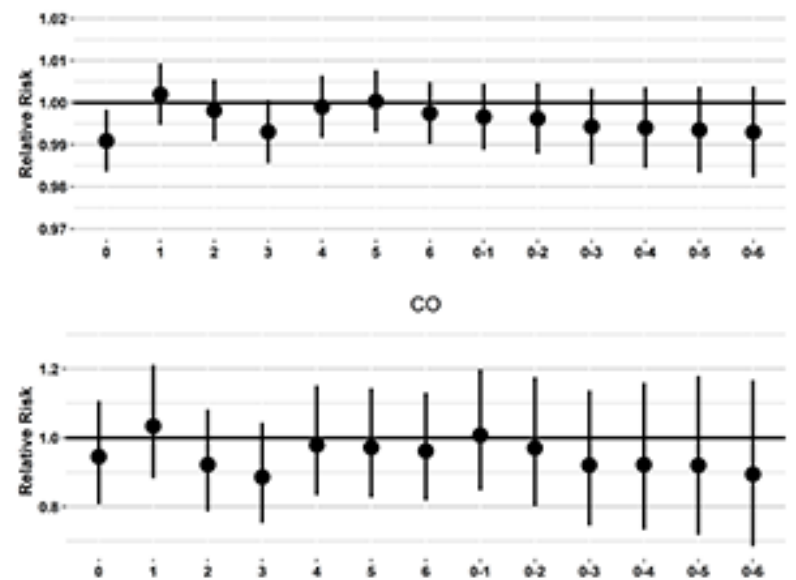

$\mathrm{PM}_{2} \mathrm{~s}$

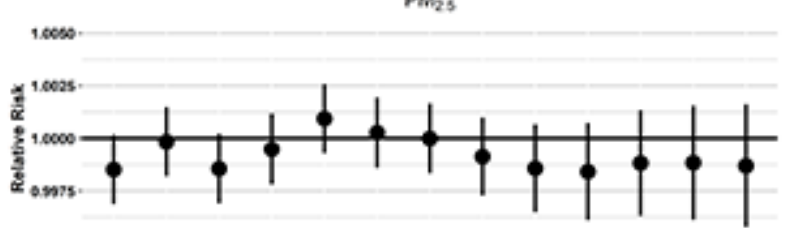

soses.
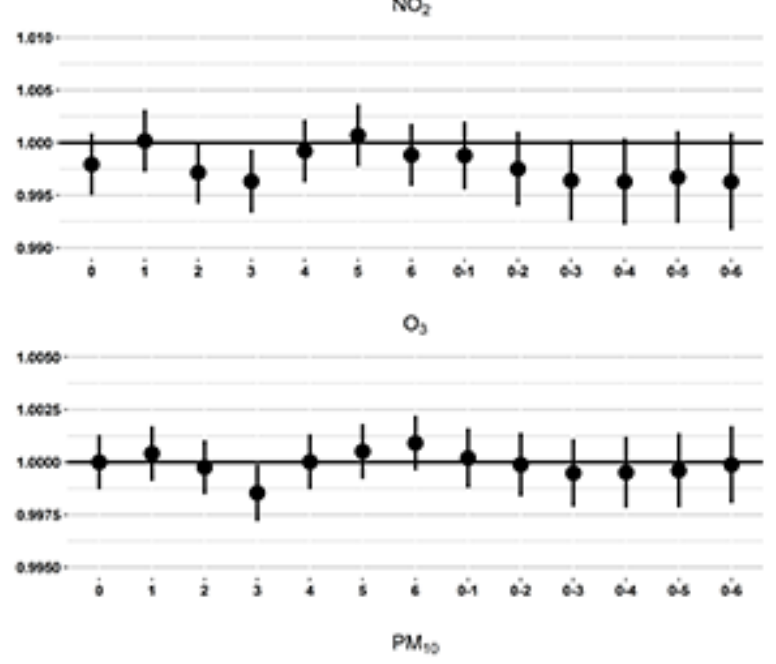

1.0000.

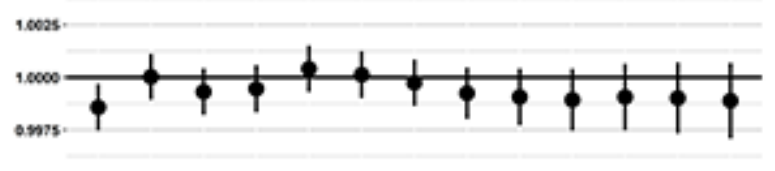

sse.

Figure 2

Page $16 / 22$ 
RR changes with $95 \%$ confidence intervals in daily asthma percentage deviations (\%). It was shown with an increase in SO2, NO2, CO, 03, PM2.5 and PM10 concentrations with different lag days in singlepollutant model.
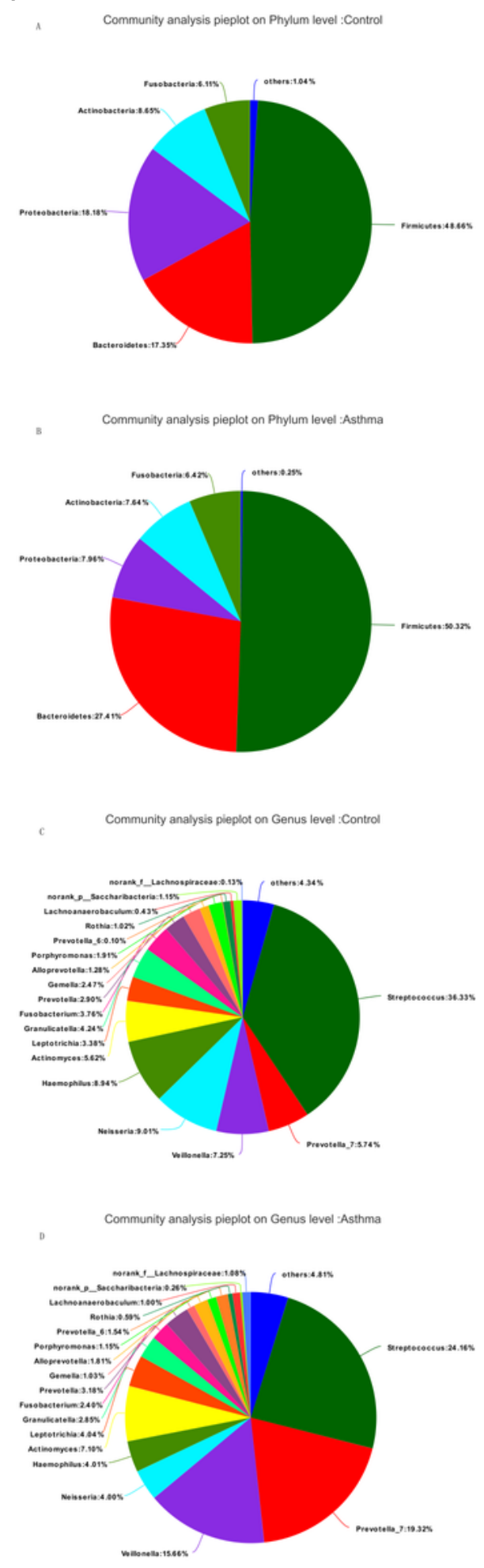

Figure 3

Microbial community compositions at phylum and genus levels. A/B at the phylum level; $C / D$ at the genus level 


\section{Prevotella}

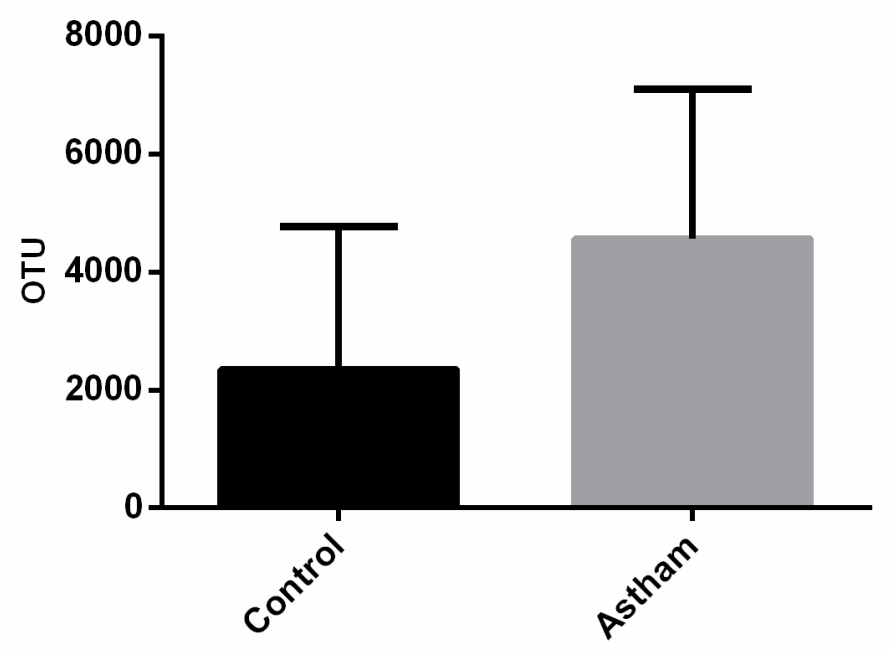

Viellonella

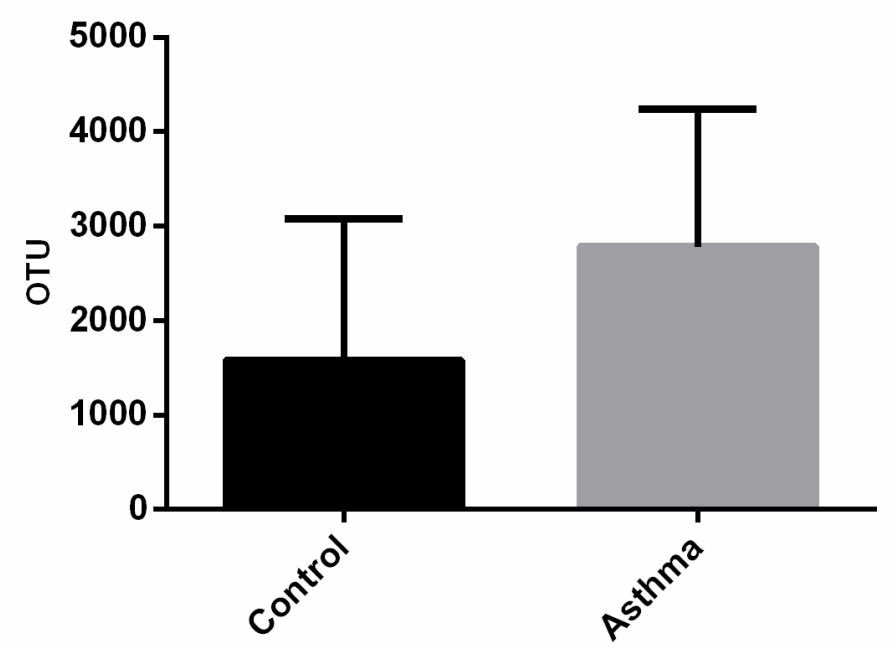

Figure 4

OTU abundance of Veillonella and Prevotella in different groups.

$\square$ Control $\square$ Asthma

Cancers

Metabolic Diseases 日

Enzyme Families

Environmental Adaptation \&

Signaling Molecules and Interaction $\mathrm{B}$

Nucleotide Metabolism

Glycan Biosynthesis and Metabolism

Signal Transduction

Digestive System ]

Lipid Metabolism

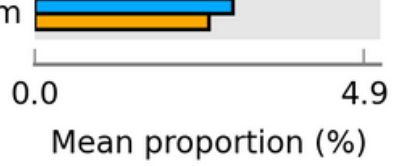

$95 \%$ confidence intervals

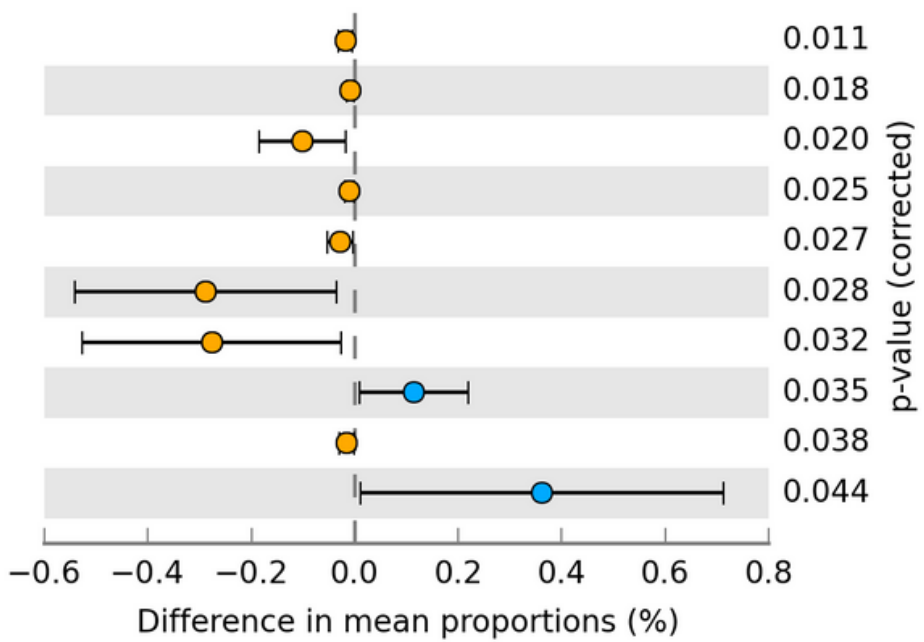

\section{Figure 5}

The different 16S rRNA OTU enriched on KEGG pathway. The enriched pathway of the metagenome based on 16S rRNA data was performed by PICRUSt. The results were presented by STAMP. Differences between groups were determined by Wilcoxon rank-sum test. 
RDA on Genus level

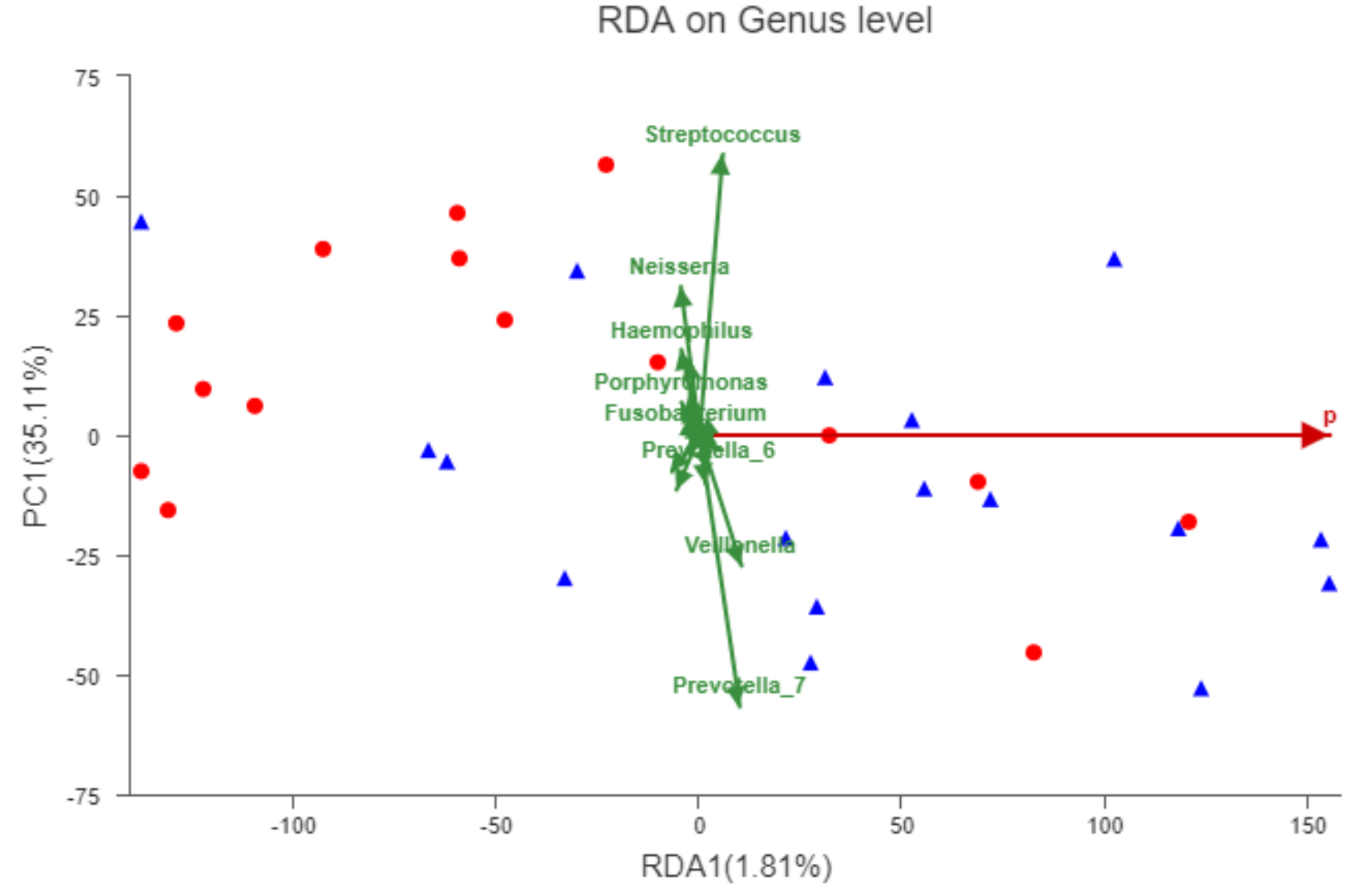

Figure 6

Redundancy analysis (RDA) plot summarizing variation in microbiota across sample properties and 1OHPyr level(p). Circles and triangles represent different group samples; red and green arrows illustrate PAHs and microbiota, respectively. Eigenvalues for the first two were presented.

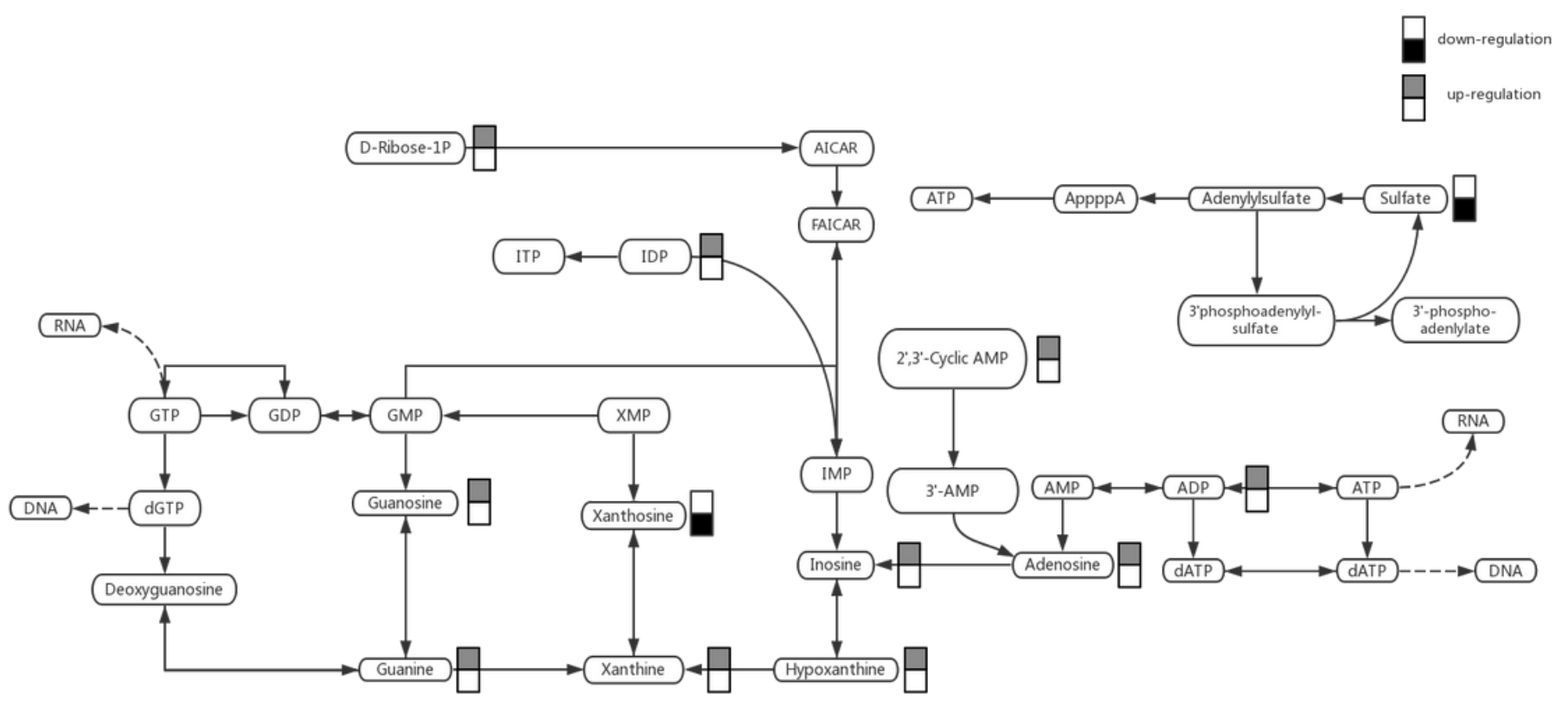




\section{Figure 7}

The differential metabolites involved in purine metabolic pathways compared with the control group. The changed metabolites are labelled for up-and down-regulation.

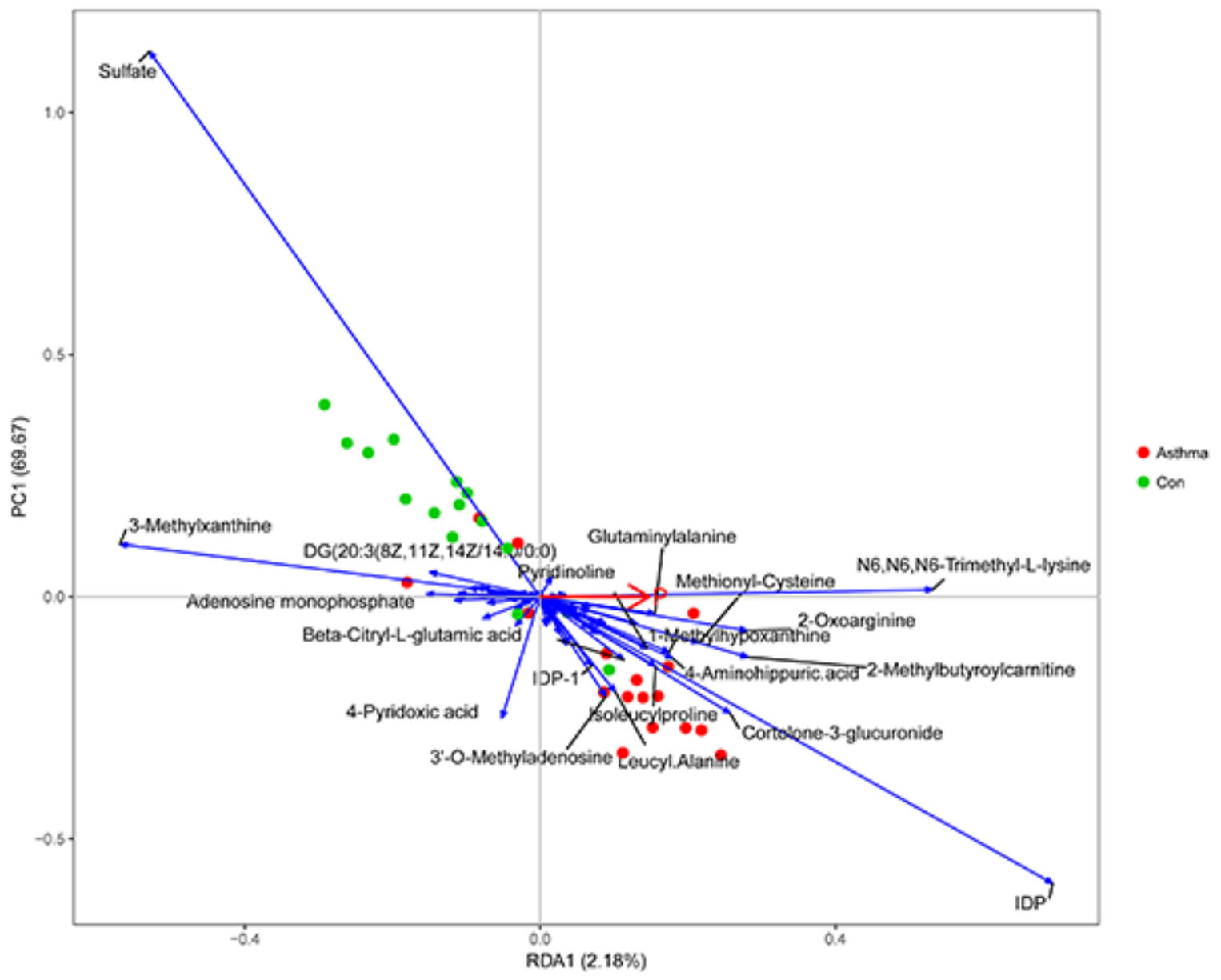

Figure 8

The RDA analysis of metabolites and 1-OHP between the control and asthmatic group. The blue arrow represented the DEMs; the red arrow represented environmental factor 1-OHP (P); the green dot was the control; the red dot was the Asthma group. The length of arrow represented the abundance of metabolites. The angel between the metabolites and 1-OHP represented the correlation. 


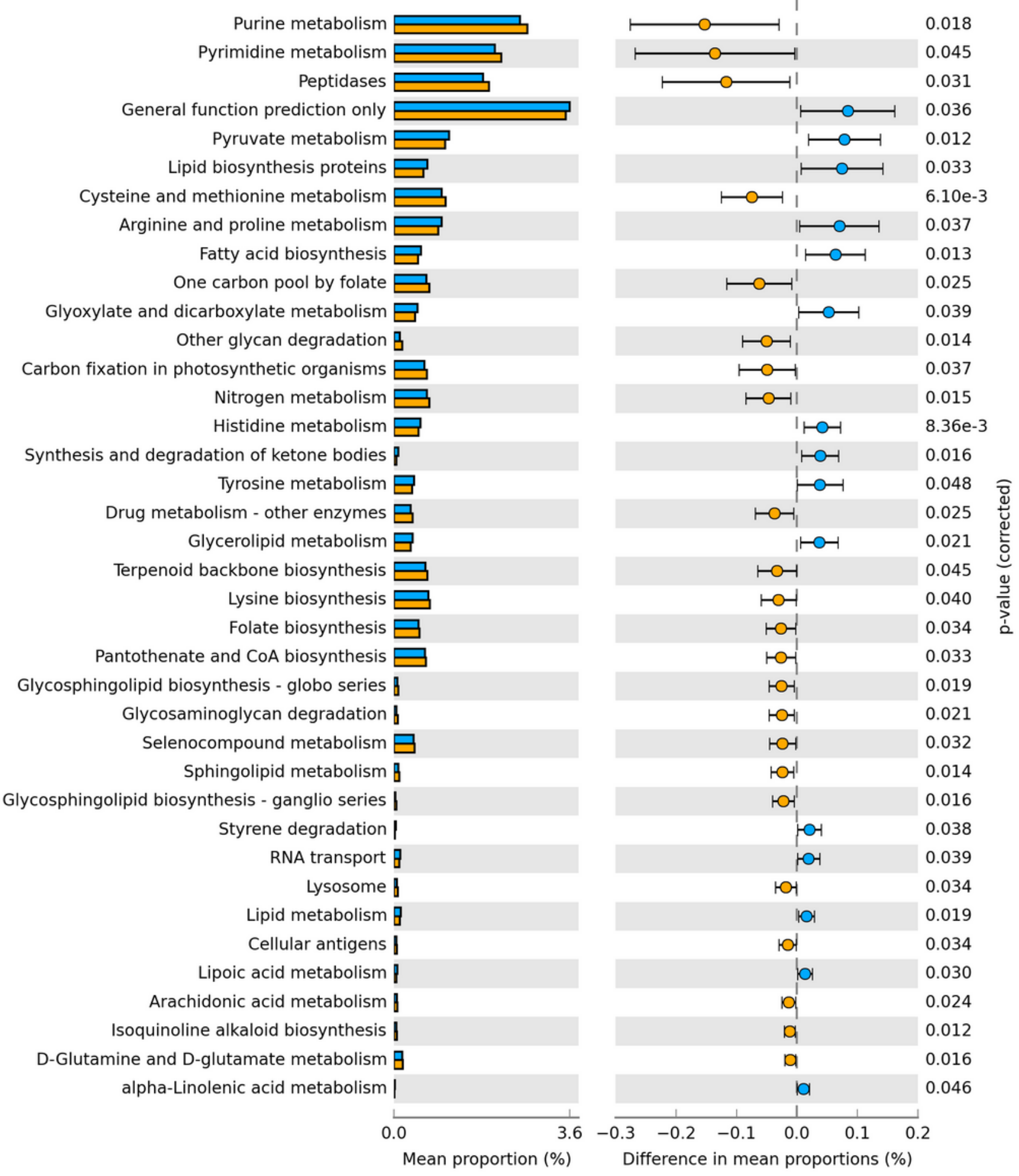

\section{Figure 9}

PAHs induced asthma by intermediating the change of microbiome and metabolome.

\section{Supplementary Files}

This is a list of supplementary files associated with this preprint. Click to download. 
- Sfig.1.tif

- Sfig.4.png

- Sfig.2.tif

- Sfig.3.tif

- Table1.docx

- STable2.docx

- STable1.docx

- STable3.docx 